\title{
ATTITUDE CONTROL SYSTEM OF THE DELFI-N3XT SATELLITE
}

\section{J. Reijneveld ${ }^{1}$ and D. Choukroun ${ }^{1,2}$}

\author{
${ }^{1}$ Faculty of Aerospace Engineering \\ Delft University of Technology (TUD) \\ Delft 2629 HS, The Netherlands \\ ${ }^{2}$ Mechanical Engineering Department \\ Ben-Gurion University of the Negev \\ Beer-Sheva 84105, Israel
}

This work is concerned with the development of the attitude control algorithms that will be implemented on board of the Delfi-n3xt nanosatellite, which is to be launched in 2013. One of the mission objectives is to demonstrate Sun pointing and three axis stabilization. The attitude control modes and the associated algorithms are described. The control authority is shared between three body-mounted magnetorquers (MTQ) and three orthogonal reaction wheels. The attitude information is retrieved from Sun vector measurements, Earth magnetic field measurements, and gyro measurements. The design of the control is achieved as a trade between simplicity and performance. Stabilization and Sun pointing are achieved via the successive application of the classical Bdot control law and a quaternion feedback control. For the purpose of Sun pointing, a simple quaternion estimation scheme is implemented based on geometric arguments, where the need for a costly optimal filtering algorithm is alleviated, and a single line of sight (LoS) measurement is required - here the Sun vector. Beyond the three-axis Sun pointing mode, spinning Sun pointing modes are also described and used as demonstration modes. The three-axis Sun pointing mode requires reaction wheels and magnetic control while the spinning control modes are implemented with magnetic control only. In addition, a simple scheme for angular rates estimation using Sun vector and Earth magnetic measurements is tested in the case of gyro failures. The various control modes performances are illustrated via extensive simulations over several orbits time spans. The simulated models of the dynamical space environment, of the attitude hardware, and the onboard controller logic are using realistic assumptions. All control modes satisfy the minimal Sun pointing requirements allowed for power generation. 


\section{INTRODUCTION}

Following up with the successful mission of its first nanosatellite, Delfi-C ${ }^{3}$, TUD is currently undertaking the development of its next satellite, Delfi-n3Xt, which three major mission objectives are

(i) three-axis active attitude control;

(ii) a single-point-of-failure free electrical power subsystem with energy storage; and

(iii) a high data rate link.

Similarly to Delfi-C ${ }^{3}$, Delfi-n3Xt is a triple-unit CubeSat that will be launched as a piggyback. Following launch and separation of the main satellite, the nanosatellite will be deployed from a Poly-Picosatellite Orbital Deployer (P-POD) on a Sun synchronous orbit at an altitude yet to be selected within a 600-1000-kilometer range.

This paper provides a partial account of the ongoing developments of the attitude control system. In particular, it presents the selection of hardware and the control modes design. The attitude control system is equipped with a 3 -axis magnetometer (MGM), a coarse Sun sensor (CSS), three body mounted rate gyros, three MTQ, developed at TUD, and three reaction wheels (RW) which spinning mass is also designed at TUD. The case of control without reaction wheels is considered.

Much research has been conducted in the field of attitude control using only magnetic information and actuation. Some works in the realm of nanosatellites are presented in [1-5]. The widely adopted Bdot control [6] allows for the detumbling of the spacecraft dynamics following the separation phase. As a result, the satellite acquires a slow rotational motion at a rate that is equal to twice its orbital motion rate. The Bdot law is further modified in order to produce a spin motion around a preferred body-axis $[1,4,5]$. The rationale for creating an additional spin motion stems from the resulting "gyroscopic rigidity" around the spin axis. It helps keeping a body axis along a preferred inertial axis, such as the LoS to the Sun along low Earth orbits (LEO) during eclipses, when the Sun sensors are not operational. The precession of the spin axis towards the Sun LoS can be achieved using Sun vector measurements and its time derivative in conjunction with the Earth magnetic field measurements [4].

It also requires an estimate of the inertial angular velocity around the spin axis. The later information can be replaced by the knowledge of the satellite angular momentum [5] but introduces a sensitivity to inertia uncertainties. In this work, two-step control strategy is adopted for the Sun acquisition and tracking. The three-axis stabilized mode implements a quaternion 
feedback control law. The quaternion vector part is estimated using the Sun vector measurement only, which is sufficient for Sun pointing purpose. As a result, the satellite is able to point towards the Sun with its two other principal axes in an undefined position. Following [4], three other modes are designed for the purpose of demonstration. A modification of the Sun coarse acquisition law is proposed here, which alleviates the strong nonlinearity due to the absolute value. Extensive simulations have been performed in order to validate the performances of the control strategies with respect to various initial conditions and angular rates. The controllers are providing pointing performances within the 20-degree bound allowed for minimal power generation. The simulations include environmental perturbation torques such as the gravity gradient, the aerodynamic drag, the solar pressure, and the residual magnetic torque.

Section 2 includes the design of the attitude control algorithms. Conclusions are presented in section 3 .

\section{ATTITUDE CONTROL SYSTEM DESIGN}

The following Cartesian coordinates frames are considered. The spacecraft body fixed frame, denoted by $\mathcal{B}$, is centered at the center of mass of the spacecraft, the $\mathcal{B}_{z}$ axis is aligned with the longitudinal axis of the spacecraft, the $\mathcal{B}_{x}$ and the $\mathcal{B}_{y}$ axes are aligned with the satellite principal axes of inertia such that the right-hand rule is respected. The MTQ and the reaction wheels rotation axis will be aligned with the body axis. The second frame of interest is a generic spacecraft centered Sun pointing coordinates frame, denoted by $\mathcal{R}$. The $\mathcal{R}_{x}$ axis is aligned with the Sun spacecraft LoS vector and is pointing from the spacecraft to the Sun. The other two axes, $\mathcal{R}_{y}$ and $\mathcal{R}_{z}$, are within the plane that is normal to $\mathcal{R}_{x}$ and respect the right-hand rule. They are fixed with respect to the first axis but the rotation angle around $\mathcal{R}_{x}$ is not specified.

As will be seen next, it is sufficient in order to design unambiguously a control law for three-axis stabilization with Sun pointing. The satellite will be Sun pointing when $\mathcal{B}_{z}$ will be in the $\mathcal{R}_{x}$ direction.

\subsection{Attitude Modes and Hardware}

The Attitude Determination and Control System (ADCS) of Delfi-n3xt will be equipped with the following sensors and actuators. Three body-mounted MGM aligned with the three axes of the spacecraft will provide onboard measurement of the Earth magnetic field. A set of six four-quadrant Sun sensors, located on each of the sides of the cubesat, will produce a continuous measurement of the 
Sun spacecraft LoS, except during the eclipses. Three body-mounted gyros will provide the inertial angular velocity of the spacecraft along the body axis. Three coils aligned along the axis of the spacecraft will be used as MTQ and produce control by interaction with the Earth magnetic field, which strength is significant enough at the heights between 600 and $1000 \mathrm{~km}$. Three reaction wheels will be used and produce additional torquing capability.

The following modes are foreseen. The detumbling mode will start right after the deployment of the satellite from the P-POD. In this mode, the rotation rates of the satellite will be reduced from their orbit insertion values down to approximately twice the orbit rotational rate. The controller will implement the standard Bdot control law [6]. Only the MGM and the MTQ will be used in this mode. From the detumbling mode, the satellite will switch to the nominal mode, where it will be 3-axis stabilized and the solar panels of the satellite will be pointing towards the Sun for maximum power generation. The control law in this mode uses proportional derivative feedback on the quaternion error and angular body rates, and is inspired from the quaternion feedback regulator introduced in [7]. It is, however, computationally less burdensome since it does not require a state estimator. All sensors and actuators will be activated in the nominal mode. For contingency reasons, a second version of the nominal mode is implemented where the angular rates are estimated from the LoS measurements rather than measured from the gyros. The above two modes constitute the sequence of the operational modes.

Additional modes are developed for demonstration purposes, when the reaction wheels are no longer available. The modified Bdot mode implements a Bdot control law in addition to magnetic torques that spin up the satellite longitudinal axis to a desired spin rate. The control law is described in [3]. Only the MGM and the MTQ will be used in this mode.

Table 1 Overview of control modes

\begin{tabular}{llc}
\hline \multicolumn{1}{c}{ Mode } & \multicolumn{1}{c}{ Sensors and actuators } & \multicolumn{1}{c}{ Pointing goal } \\
\hline Detumble mode & Magnetometers and MGQ & Reduce rotational rates \\
\hline Nominal mode & $\begin{array}{l}\text { Magnetometers, gyroscopes, } \\
\text { Sun sensors, MGQ, and re- } \\
\text { action wheels }\end{array}$ & Sun pointing \\
\hline \multirow{2}{*}{ Back-up Nominal mode } & $\begin{array}{l}\text { Magnetometers, Sun sen- } \\
\text { sors, MTQ, and reaction }\end{array}$ & Sun pointing \\
& wheels & \\
\hline Modified Bdot mode & Magnetometers and MTQ & Controlled spin around $z$ axis \\
\hline FSP mode & $\begin{array}{l}\text { Magnetometers, Sun sen- } \\
\text { sors, and MTQ }\end{array}$ & \\
\hline CSP mode & Magnetometers, Sun stabilized Sun pointing \\
& sors, and MTQ & Spin stabilized Sun pointing \\
\hline
\end{tabular}


Upon reaching the desired spin rate, the satellite will enter the fine Sun pointing (FSP) mode and point its solar panels towards the Sun using a nonlinear control with only the MTQ and LoS and angular rates information. The control is described in $[1,4]$. The MGM, the Sun sensors, the MTQ, and the gyros will be used.

An additional demonstration mode is the coarse Sun pointing (CSP), where the solar panels will point towards the Sun using a nonlinear control law with only LoS information $[1,4]$. The MGM, the Sun sensors, and the MTQ will be used.

An overview of the control modes and the used hardware is given in Table 1.

\subsection{Control Algorithms}

\subsubsection{Detumbling}

In the detumbling mode, the controller applies the standard Bdot control law [6]. The Earth magnetic field, $\mathbf{b}$, is continuously measured onboard the satellite, and its time derivative, $\dot{\mathbf{b}}$, is computed via a forward difference scheme. The magnetorquers will be turned on in order to produce a magnetic dipole that is proportional to $\dot{\mathbf{b}}$. As a result, the satellite rotational rates will decrease until they reach values of the order of twice the orbit rotational rate. The control law is described as follows:

$$
\mathbf{m}_{\text {mag }}=-\kappa_{\text {mag }} \dot{\mathbf{b}}
$$

where $\mathbf{m}_{\text {mag }}$ denotes the magnetic dipole and $\kappa_{\text {mag }}$ is the positive gain parameter chosen such that the MTQ are saturated until the rotational rate has been lowered significantly; so, it functions as a bang-bang controller. From that point on a smooth reduction of the rotational rate to twice, the orbital rate is reached. The resulting magnetic control torque is as follows:

$$
\mathbf{T}_{\text {mag }}=\mathbf{m}_{\text {mag }} \times \mathbf{b} .
$$

This control law will be used until the value of $\dot{b}_{z}$ remains below a predefined limit - here, $10^{-7} \mathrm{~T} / \mathrm{s}$, for a certain amount of time - here, one orbit. Care has to be taken when setting this limit in order not to exit the detumbling mode prematurely.

\subsubsection{Nominal mode - three-axis stabilized Sun pointing}

In the nominal mode, the satellite is three-axis stabilized with $\mathcal{B}_{z}$, the longitudinal axis, pointing towards the Sun. The control torque is designed following the proportional-derivative approach introduced in [7]: 


$$
\mathbf{T}_{\mathrm{des}}=-\kappa_{\omega} J \boldsymbol{\omega}_{e}-\kappa_{q} J \mathbf{e}_{e}
$$

where $\kappa_{\omega}$ and $\kappa_{q}$ are the scalar gain parameters, selected such that the desired orientation is reached within a fraction of the orbital period; and $J$ denotes the symmetric tensor of inertia of the spacecraft with respect to the principal axis. The vector $\boldsymbol{\omega}_{e}$ denotes the tracking error in the body components of the inertial angular velocity and the vector $\mathbf{e}_{e}$ denotes the tracking error in the vector part of the quaternion of rotation from $\mathcal{B}$ to $\mathcal{R}$. In the following, two cases of angular rates information will be considered. In the first case, the vector $\boldsymbol{\omega}_{e}$ is measured as the output of a triad of body-mounted gyros. In the second case, this vector is estimated based on a continuous Sun vector measurement history. The vector $\mathbf{e}_{e}$ is estimated using the Sun vector LoS only. These estimation algorithms are described in the ensuing. The torque $\mathbf{T}_{\text {des }}$ denotes the total required torque, which can effectively be delivered via simultaneous actuation of the reaction wheels and of the MTQ. In this work, the control allocation is implemented by the geometrical approach presented in [8]. The desired torque is decomposed into two perpendicular components, where the first one, $\mathbf{T}_{\mathrm{rw}}$ is parallel to the Earth magnetic field and the second one, $\mathbf{T}_{\text {mag }}$, is perpendicular to it. The control allocation is thus simple: the MTQ will apply the component $\mathbf{T}_{\text {mag }}$, while the reaction wheels will handle the remainder. The required magnetic dipole, denoted as $\mathbf{m}_{\mathrm{mag}}$ is calculated as follows:

$$
\mathbf{m}_{\mathrm{mag}}=-\frac{\mathbf{T}_{\mathrm{des}} \times \mathbf{b}}{|\mathbf{b}|^{2}} .
$$

As a result, the torque that is required from the reaction wheels is:

$$
\mathbf{T}_{\mathrm{rw}}=\mathbf{T}_{\mathrm{des}}-\mathbf{m}_{\mathrm{mag}} \times \mathbf{b} .
$$

In this work, it is assumed that the reaction wheels can fully apply $\mathbf{T}_{\mathrm{rw}}$.

\subsubsection{Estimation of the vector $\mathrm{e}_{e}$}

This paragraph introduces a method for estimation of the attitude vector $\mathbf{e}_{e}$, which is computationally very simple, only requires the Sun vector measurement, and yet fits the requirement of designing a Sun pointing control law via Eq. (1). In order to have the satellite in a Sun pointing attitude, the only requirement is that the $\mathcal{B}_{z}$ axis is Sun pointing. Let $\mathbf{s}=\left[s_{x}, s_{y}, s_{z}\right]^{\mathrm{T}}$ denote the $\mathrm{S} / \mathrm{C}$-sun unit vector in $\mathcal{B}$, and $\theta$ denote the coelevation of the Sun unit vector in $\mathcal{B}$, i. e.,

$$
\tan \theta=\frac{\sqrt{s_{x}^{2}+s_{y}^{2}}}{s_{z}} .
$$


It is straightforward to see that $\theta$ is the angle of the smallest rotation that brings the $\mathcal{B}_{z}$ axis onto the Sun unit vector. The unit vector axis of this rotation is simply expressed as follows:

$$
\hat{\mathbf{u}}=\frac{1}{\sqrt{s_{x}^{2}+s_{y}^{2}}}\left[\begin{array}{c}
s_{y} \\
-s_{x} \\
0
\end{array}\right] .
$$

The quaternion of this rotation can be easily expressed as a function of $\theta$ and $\hat{u}$. In particular, the vector part, $\mathbf{e}_{e}$, is expressed as

$$
\mathbf{e}_{e}=\sin \left(\frac{\theta}{2}\right) \hat{\mathbf{u}}
$$

where $\theta$ and $\hat{\mathbf{u}}$ are provided in Eqs. (2) and (3) as a function of the Sun vector component along $\mathcal{B}$. For small values of $\theta$, the vector $\mathbf{e}_{e}$ can be approximated, i. e.,

$$
\mathbf{e}_{e} \sim \frac{1}{2}\left[\begin{array}{c}
\frac{s_{y}}{s_{z}} \\
-\frac{s_{x}}{s_{z}} \\
0
\end{array}\right] .
$$

\subsubsection{Estimation of the angular velocity vector}

The primary implementation of the control law is by using the angular velocity as measured by the gyros. In this work, only white noise is considered. Gyro biases can, however, be estimated using standard techniques. This paragraph describes a method that estimates the angular velocity vector from LoS measurements [3]. Let $\mathbf{b}$ and $\mathbf{s}$ denote the unit Earth magnetic field and unit Sun vector measurements along the frame $\mathcal{B}$. First, compute

$$
\begin{aligned}
& \boldsymbol{\omega}_{b \perp}=-\mathbf{b} \times \dot{\mathbf{b}} ; \\
& \boldsymbol{\omega}_{s \perp}=-\mathbf{s} \times \dot{\mathbf{s}} .
\end{aligned}
$$

Then, compute the following estimates of $\boldsymbol{\omega}$ :

$$
\begin{aligned}
& \hat{\boldsymbol{\omega}}_{1}=\boldsymbol{\omega}_{B \perp}+\frac{\left(\boldsymbol{\omega}_{S \perp}-\boldsymbol{\omega}_{B \perp} \times \mathbf{s}\right)^{\mathrm{T}}(\mathbf{b} \times \mathbf{s})}{|\mathbf{b} \times \mathbf{s}|^{2}} \mathbf{b} ; \\
& \hat{\boldsymbol{\omega}}_{2}=\boldsymbol{\omega}_{S \perp}+\frac{\left(\boldsymbol{\omega}_{S \perp}-\boldsymbol{\omega}_{B \perp} \times \mathbf{b}\right)^{\mathrm{T}}(\mathbf{b} \times \mathbf{s})}{|\mathbf{b} \times \mathbf{s}|^{2}} \mathbf{s} .
\end{aligned}
$$

Finally, an estimate is given as the mean of the previous ones:

$$
\hat{\boldsymbol{\omega}}=\frac{\hat{\boldsymbol{\omega}}_{1}+\hat{\boldsymbol{\omega}}_{2}}{2} \text {. }
$$


This estimated inertial angular velocity of $\mathcal{B}$ is used when gyros measurements are not available. It is emphasized that here, $\mathbf{b}$ denotes the unitized measured Earth magnetic field.

\subsubsection{Wheels desaturation strategy}

Once the angular momentum stored in one of the reaction wheels reaches $5 \%$ of the maximum, the MTQ are activated and allow for the wheels to desaturate down to $1 \%$ of their maximum. This is done without impairing the pointing attitude of the satellite. The torque that is required for desaturation, $\mathbf{T}_{\mathrm{rw} U R}$, is calculated from the current angular momentum stored in the reaction wheels. The magnetic dipole, $\mathbf{m}_{\mathrm{rw} U}$, is thus obtained as follows:

$$
\mathbf{m}_{\operatorname{mag} U}=-\frac{\mathbf{T}_{\mathrm{rw} U R} \times \mathbf{b}}{|\mathbf{b}|^{2}} .
$$

Since, for desaturation purposes, the direction of the magnetic torque is important, rather than its magnitude, the vector $\mathbf{m}_{\mathrm{rw} U}$ is scaled to have a maximal length. Thus, the magnetic torque is stronger and the desaturation effect is more efficient:

$$
\mathbf{m}_{\mathrm{mag} U}^{*}=m_{\mathrm{MAG}} \frac{\mathbf{m}_{\mathrm{mag} U}}{\left\|\mathbf{m}_{\operatorname{mag} U}\right\|}
$$

where $m_{\mathrm{MAG}}$ denotes the maximal available dipole for the given MTQ. In order to leave the attitude of the satellite unchanged, the magnetic torque produced by $\mathbf{m}_{\operatorname{mag} U}^{*}$ is being continuously canceled by the reaction wheels producing a torque $\mathbf{T}_{\text {rw } U}$ that is equal in magnitude and opposite in direction, thus allowing them to desaturate:

$$
\mathbf{T}_{\mathrm{rw} U}=-\mathbf{m}_{\operatorname{mag} U}^{*} \times \mathbf{b} .
$$

The above desaturation strategy is applied continuously which is good for low power consumption. Indeed, desaturating the wheels at lower velocities requires less torque and thus less power than at higher velocity. Other unloading strategies will, however, have to be investigated in view of the trade between power consumption and reaction wheel wear and tear.

\subsubsection{Modified Bdot}

In this demonstration mode, the controller will bring the satellite to a desired spin rate around the $z$-axis using only MGM measurements and the MTQ. The control law is given by [4] as follows:

$$
\mathbf{m}_{\mathrm{mag}}=-\kappa_{\mathrm{mag}}\left(\dot{\mathbf{b}}+\boldsymbol{\omega}_{d} \times \mathbf{b}\right)
$$


where $\mathbf{m}_{\text {mag }}$ denotes the required magnetic dipole; $\kappa_{\text {mag }}$ is the positive scalar gain chosen again to saturate the wheels until the nominal desired rotational rate is almost reached; $\mathbf{b}$ is the measured magnetic field; and $\boldsymbol{\omega}_{d}$ is the desired spin rate around the $z$-axis of the body frame. All vectors are projected in the body reference frame and the time derivative of the magnetic field, $\dot{\mathbf{b}}$, is computed using a forward difference scheme. As in the simple Bdot mode, this control law will be used until the value of $\dot{\mathbf{b}}_{z}$ remains below a predefined limit for a certain amount of time - here, below $10^{-7} \mathrm{~T} / \mathrm{s}$ during one orbit. Notice that the other components of $\dot{\mathbf{b}}$ will keep changing, since the satellite will be spinning around the $z$-axis.

\subsubsection{Coarse Sun pointing}

When the satellite has reached the desired spin up rate and the Sun vector measurements are available, the satellite enters the coarse Sun pointing mode. This mode augments the modified Bdot controller by using the $\mathcal{B}_{z}$ axis MTQ in order to point it toward the Sun. Since this MTQ only produces torques in the $x-y$ plane, the dot product between the torque $\mathbf{T}$ produced by the $z \mathrm{MTQ}$ and the $x$ and $y$ parts of the Sun vector $\mathbf{s}$ will approach zero when the $z$-axis is pointing towards the Sun:

$$
\mathbf{T}^{\mathrm{T}} \mathbf{s}=(\mathbf{m} \times \mathbf{b})^{\mathrm{T}} \mathbf{s}=m_{z}\left(b_{x} s_{y}-b_{y} s_{x}\right)=0 .
$$

Thus, the quantity $b_{x} s_{y}-b_{y} s_{x}$ is used as an error signal in the control law. The commanded magnetic dipole is given as

$$
\mathbf{m}_{\operatorname{mag} z}=\kappa_{C}\left(b_{x} s_{y}-b_{y} s_{x}\right)
$$

where $\kappa_{\text {mag }}$ is the positive gain, which should be carefully selected in order not to interfere with the Bdot control and not to produce a too large overshoot. This can be prevented by utilizing a gain weighter of the form $\left(1-s_{z}\right)$, which approaches zero as the satellite becomes Sun pointing. The total commanded dipole, including the Bdot controller dipole, is then

$$
\mathbf{m}=-\kappa_{\operatorname{mag}} \dot{\mathbf{b}}+\left[\begin{array}{c}
0 \\
0 \\
\left(1-s_{z}\right) \kappa_{C}\left(b_{x} s_{y}-b_{y} s_{x}\right)
\end{array}\right] .
$$

Through the weighting factor, $\left(1-s_{z}\right)$, this control law is a modification from the control law introduced in $[1,4]$.

\subsubsection{Fine Sun pointing}

Once CSP is achieved, the satellite enters the FSP mode. In this mode, the time derivative of the Sun vector and the estimated angular rates are needed. Time 
differentiation is performed by forward difference computations. The angular velocity vector is measured using the gyros. Another option is to use the angular velocity estimation algorithm described earlier, which is based on the Sun vector and the Earth magnetic field measurements. Yet, an additional approach might be used to modify the method in [9] and to assume that the magnetic field vector and the Sun vector are constant in the inertial frame. The gyro-based and estimation-based angular rates are implemented in this work. This control law was proposed in $[1,3,4]$. To maintain the desired spin rate around the $\mathcal{B}_{z}$ axis, the following torque is needed:

$$
T_{z}=\kappa_{F}\left(\omega_{d_{z}}-\hat{\omega}_{z}\right)
$$

where $\kappa_{F}$ is a positive scalar gain similar in size to the CSP mode gain; $\omega_{d_{z}}$ denotes the desired spin rate; and $\hat{\omega}_{z}$ denotes the estimated spin rate around the $z$ axis. This torque can be converted to a desired dipole with

$$
\left[\begin{array}{l}
m_{F_{x}} \\
m_{F_{y}}
\end{array}\right]=\frac{T_{z}}{b_{x}^{2}+b_{y}^{2}}\left[\begin{array}{c}
b_{y} \\
-b_{x}
\end{array}\right] .
$$

Just as in the CSP controller, the $z$ MGQ is used for precession and nutation control. In this controller, the $x$ and $y$ components of the Sun vector will be driven to zero. The torque is given by

$$
\mathbf{T}=\kappa_{F_{p}} \hat{\omega}_{z}\left[\begin{array}{c}
s_{x} \\
s_{y} \\
0
\end{array}\right]-\kappa_{F_{d}}\left[\begin{array}{c}
\hat{\omega}_{z} s_{x}+\dot{s}_{y} \\
\hat{\omega}_{z} s_{y}+\dot{s}_{x} \\
0
\end{array}\right]
$$

where $\kappa_{F_{p}}$ and $\kappa_{F_{d}}$ are the positive scalar gains. From this equation, the magnetic dipole of the $z$ MGQ can be calculated as

$$
m_{F_{z}}=\frac{b_{x} T_{y}-b_{y} T_{x}}{b_{x}^{2}+b_{y}^{2}} .
$$

\section{SIMULATION}

\subsection{Description}

Extensive simulations were run to test the proposed control design. The dynamic model used in the simulation of the satellite equations of motion is as follows:

$$
J \dot{\boldsymbol{\omega}}=-\Omega\left(J \boldsymbol{\omega}+\mathbf{h}_{\mathrm{rw}}\right)-\dot{\mathbf{h}}_{\mathrm{rw}}+\mathbf{T}_{\mathrm{mag}}+\mathbf{T}_{\mathrm{gg}}+\mathbf{T}_{d_{\mathrm{mag}}}+\mathbf{T}_{\mathrm{drag}}+\mathbf{T}_{\mathrm{sol}}
$$


Table 2 Simulation parameters

\begin{tabular}{lc}
\hline \multicolumn{1}{c}{ Parameter } & Value \\
\hline Inclination & $97.8^{\circ}$ \\
Altitude & $600 \mathrm{~km}$ \\
Eccentricity & 0 \\
Right ascension ascending node & $0^{\circ}$ \\
Magnetic field & $\mathrm{IGRF} 11$ \\
Inertia principal moments & $0.037,0.037,0.006 \mathrm{~kg} \cdot \mathrm{m}^{2}$ \\
Atmospheric drag torque & $2.27 \cdot 10^{-7} \mathrm{~N} \cdot \mathrm{m}$ \\
Magnetic disturbance dipole & $0.001 \mathrm{~A} \cdot \mathrm{m}^{2}$ \\
Solar disturbance torque & $6.82 \cdot 10^{-9} \mathrm{~N} \cdot \mathrm{m}$ \\
\hline
\end{tabular}

where $\mathbf{T}_{\mathrm{gg}}$ denotes the gravity gradient disturbance torque; $\mathbf{T}_{d_{\mathrm{mag}}}$ denotes the disturbance magnetic torque; $\mathbf{T}_{\text {sol }}$ denotes the solar disturbance torque; and $\mathbf{T}_{\text {drag }}$ denotes the disturbance atmospheric torque. The gravity gradient torque expression is as follows:

$$
\mathbf{T}_{\mathrm{gg}}=3 n^{2}\left[\begin{array}{ccc}
0 & -C_{33} & C_{23} \\
C_{33} & 0 & -C_{13} \\
-C_{23} & C_{13} & 0
\end{array}\right] J\left[\begin{array}{l}
C_{13} \\
C_{23} \\
C_{33}
\end{array}\right]
$$

where $C_{13}, C_{23}$, and $C_{33}$ are the body components of the unit vector pointing from the spacecraft towards the center of Earth, also known as nadir vector. The magnetic control $\mathbf{T}_{\text {mag }}$ and disturbance $\mathbf{T}_{d_{\mathrm{mag}}}$ torques are computed as follows:

$$
\begin{aligned}
& \mathbf{T}_{\mathrm{mag}}=\mathbf{m}_{\mathrm{mag}} \times A_{\mathcal{B}}^{\mathrm{IGRF}} \mathbf{r} ; \\
& \mathbf{T}_{d_{\mathrm{mag}}}=\mathbf{m}_{d_{\mathrm{mag}}} \times A_{\mathcal{B}}^{\mathrm{IGRF}} \mathbf{r}
\end{aligned}
$$

where $A_{\mathcal{B}}^{\text {IGRF }}$ denotes the rotation matrix from the IGRF frame to the body frame and $\mathbf{r}$ is the Earth magnetic along the IGRF axis. Using the Earth magnetic field in the IGRF reference frame, the solar and drag disturbance torques are simulated assuming constant magnitude and random directions. The kinematics equations are simulated using the quaternion of rotation, $\mathbf{q}$ :

$$
\dot{\mathbf{q}}=\frac{1}{2} \Omega_{4} \mathbf{q} \text {. }
$$

Here,

$$
\Omega_{4}=\left[\begin{array}{cc}
-[\boldsymbol{\omega} \times] & \boldsymbol{\omega} \\
-\boldsymbol{\omega}^{\mathrm{T}} & 0
\end{array}\right]
$$

where $\boldsymbol{\omega}$ denotes the true inertial angular velocity of the body frame along the body frame. Simulation numerical parameters are given in Table 2. Table 3 shows the parameters used for the attitude system hardware models. 
Table 3 Sensors and actuators parameters

\begin{tabular}{lc}
\hline \multicolumn{1}{c}{ Parameter } & Value \\
\hline Magnetometer bias & $300 \mathrm{nT}$ \\
Magnetometer noise & $200 \mathrm{nT}$ \\
Sun sensor bias & $2^{\circ}$ \\
Sun sensor noise & $3^{\circ}$ \\
Gyro noise & $0.06 \mathrm{deg} / \mathrm{s}$ \\
Magnetorquer maximum dipole & $0.05 \mathrm{~A} \cdot \mathrm{m}^{2}$ \\
Reaction wheel maximum torque & $10^{-5} \mathrm{~N} \cdot \mathrm{m}$ \\
Reaction wheel maximum angular momentum & $7.8 \cdot 10^{-4} \mathrm{~N} \cdot \mathrm{m} \cdot \mathrm{s}$ \\
\hline
\end{tabular}

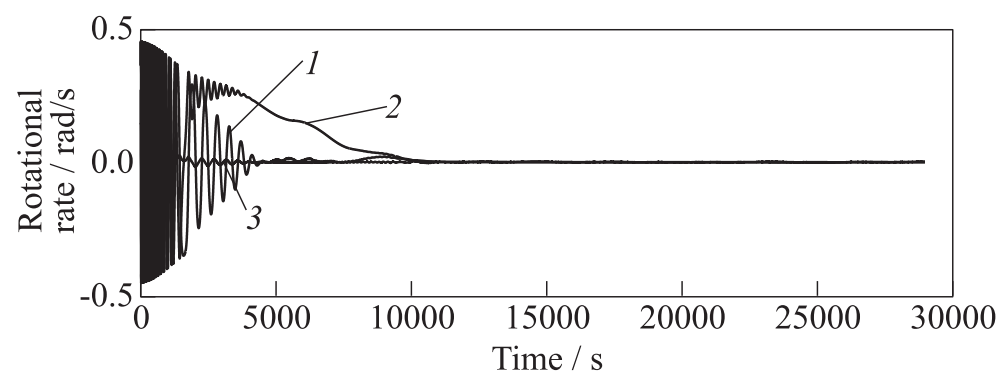

(a)

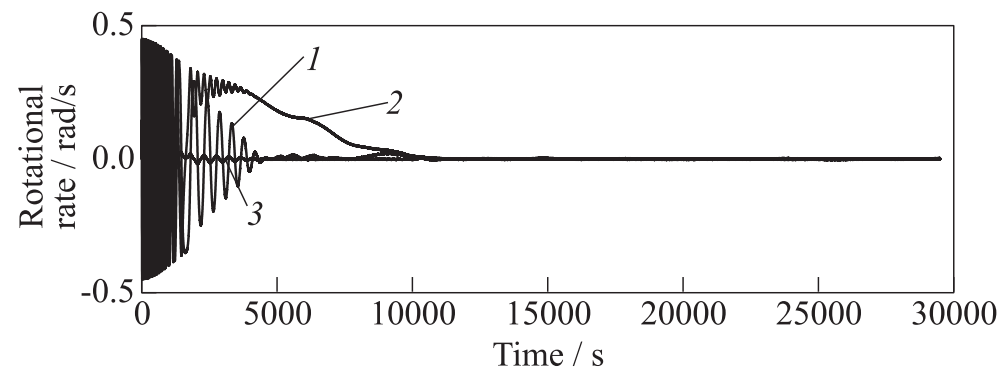

(b)

Figure 1 Detumble mode; the rates are damped within two orbits starting from high initial rotational rates: $(a)$ body rotational rates with regard to inertial frame; (b) estimated body rates; $1-w_{x} ; 2-w_{y}$; and $3-w_{z}$ 
The control algorithms developed in this paper will be implemented in the microcontroller unit (MCU) of the satellite. In order to simulate the behavior of the satellite as close as possible to the onboard behavior, the following activity sequence of the MCU has been implemented in the simulation.

1. Receive data from onboard computer. For example, new parameters, desired control mode, desired determination mode.

2. Turn off the MTQ in order to perform clean magnetic measurements.

3. Perform two consecutive magnetic measurements (or more) and store the average.

4. Calculate the time derivative of the magnetic field using forward differences.

5. Perform measurements for the remaining sensors: Sun sensors and gyros. A coarse attitude and rotational rate can be estimated in this step.
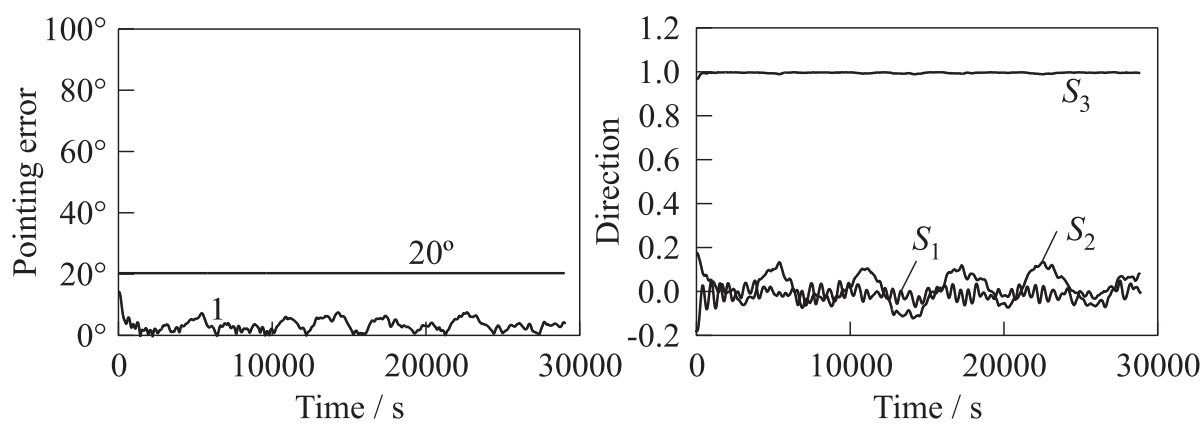

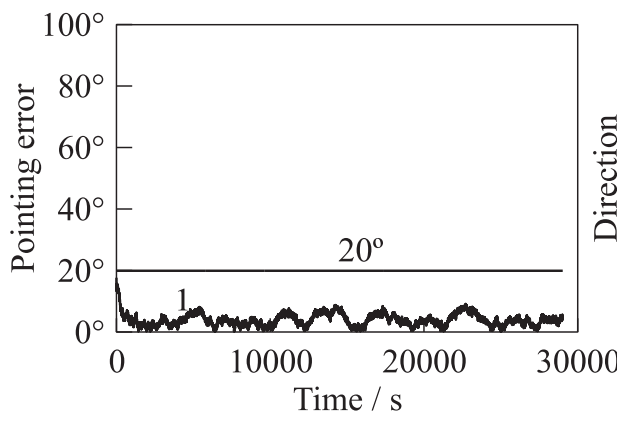

(a)

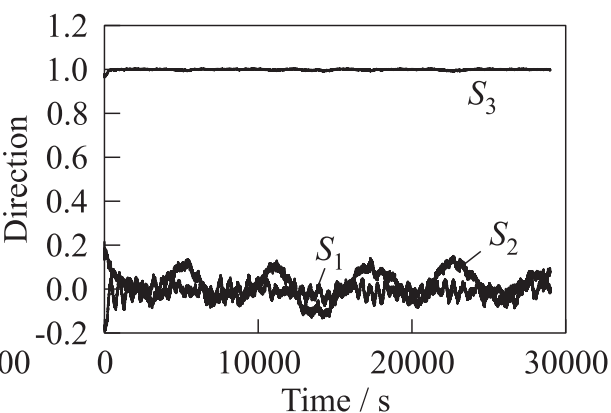

(b)

Figure 2 Nominal mode; Sun pointing is achieved starting from low initial pointing errors 
6. Activate the desired control algorithm. The desired control algorithm is determined by internal processes in the $M C U$ or by the onboard computer. The standard mode is the detumbling mode. According to the active control mode, the required control torque is calculated. Then, the MTQ and possibly the reaction wheels are activated. Unloading of the reaction wheels also takes place in this step. If attitude determination is needed for the control algorithm, the attitude determined in the previous cycle will be used.

7. Determine the current attitude. This step might take multiple cycles. The process will, therefore, be interrupted to start with a new cycle, and continued until the attitude has been determined. This part will not be explained in this paper.

8. Prepare housekeeping data to be sent back to the onboard computer. For example, sensor data, control algorithm data, determination algorithm data.
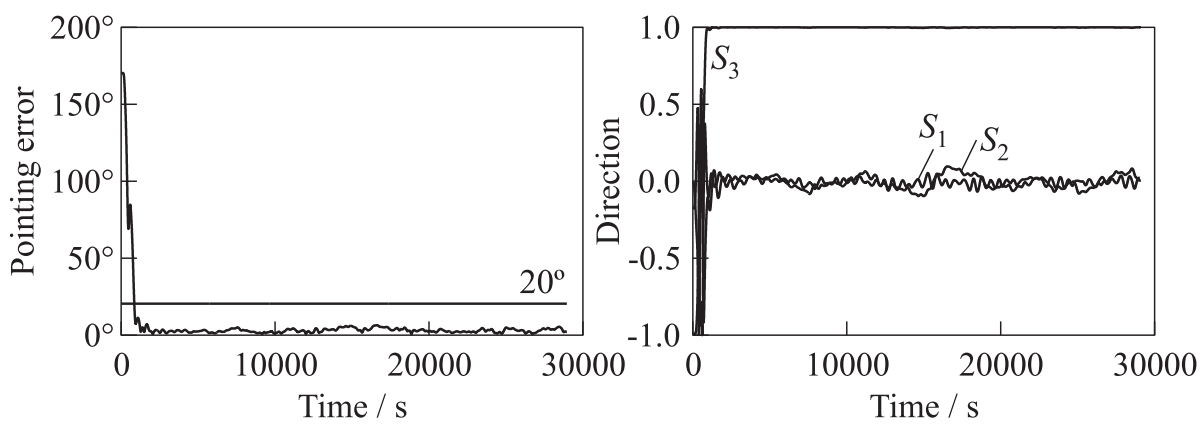

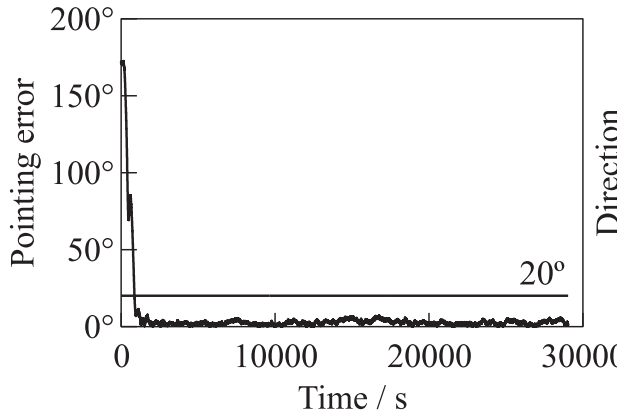

(a)

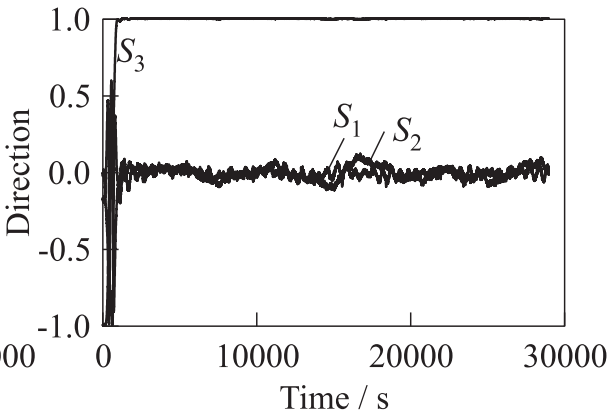

(b)

Figure 3 Nominal mode; Sun pointing is achieved starting from high initial pointing errors 


\subsection{Results}

The simulation runs are performed over $30,000 \mathrm{~s}$ which is approximately the time laps of 6 orbits of the satellite around Earth.

Figure $1 a$ depicts the true angular rates along the body axis. This shows that, during the detumble mode, high initial angular rates (about $30 \mathrm{deg} / \mathrm{s}$ ) are damped down to nearly zero within one to two orbits.

For illustration purpose, Fig $1 b$ shows the estimates of the rates as computed using the Sun vector and Earth magnetic field measurements. These estimates are clearly close to the actual values.

Figure 2 shows the performances of the nominal mode in the case of small initial pointing errors.

Figure $2 a$ shows the pointing error histories, as computed using the true values in the upper graph and computed (onboard) using the estimated values of the Sun vector and satellite's attitude, in the lower graph.

Figure $2 b$ depicts the time histories of the body components of the Sun vector. The upper graph shows the true values and the lower graph shows the estimated
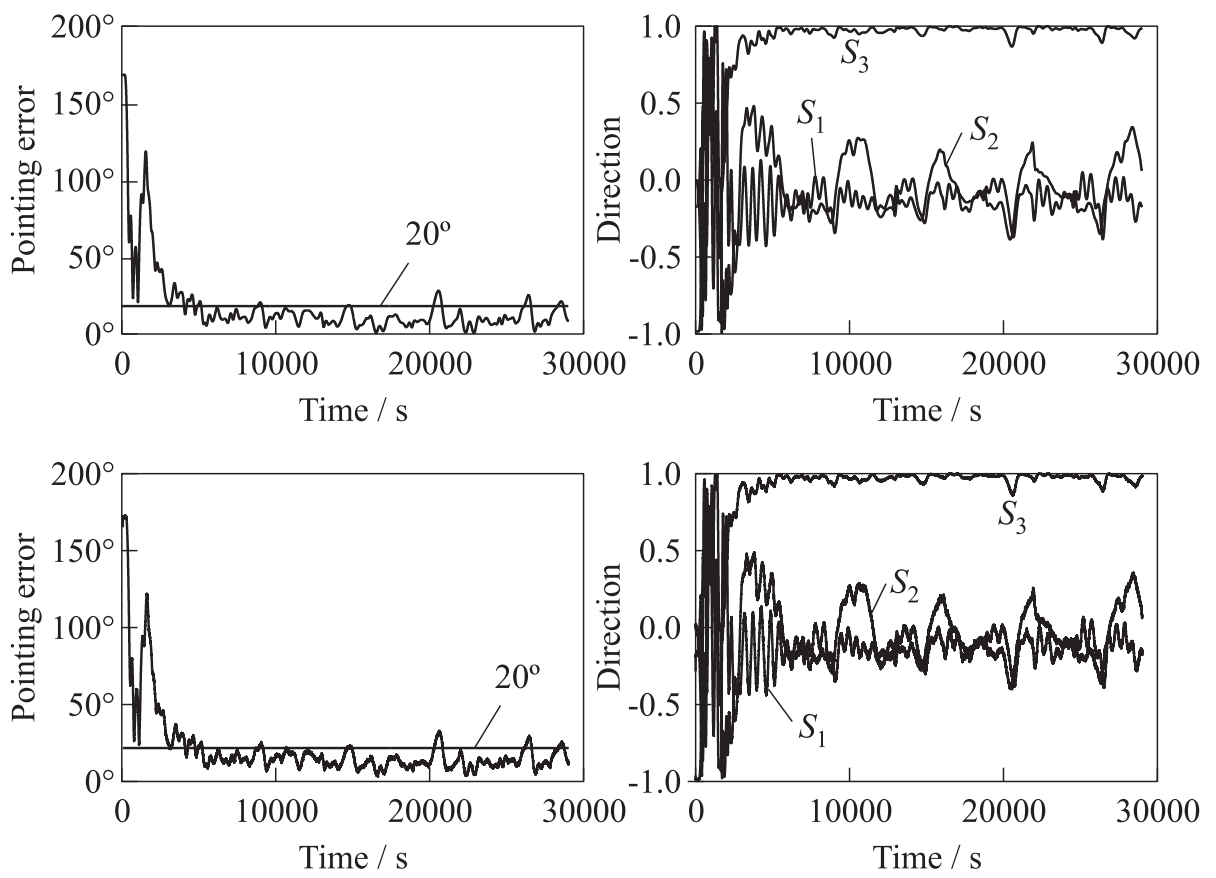

Figure 4 Nominal mode; Sun pointing is achieved using estimated angular rates 
values. As a result, the nominal mode controller is able to keep the satellite Sun pointing within the required pointing accuracy of $20^{\circ}$.

Figure 3 shows analogue results starting with very high initial pointing errors; here, $180^{\circ}$. In both cases, the quaternion estimation scheme was implemented in the feedback control.

When gyro measurements are not available, the angular rates need to be estimated using the two vector measurements. Starting with a 180-degree pointing error and using the estimated angular rates yields the pointing performances are shown in Fig. 4. When comparing this with Fig. 3, one can clearly see that the performance has decreased and the required 20-degree Sun pointing is not reached in each situation. As this is back up in case of gyroscope failure, this performance is, however, still acceptable.

Figure 5 illustrates the efficiency of the modified Bdot control law: within two orbits, the angular rates are acquiring the spin along the $z$ axis, here $3 \mathrm{deg} / \mathrm{s}$, and are close to zero in the other two axis.

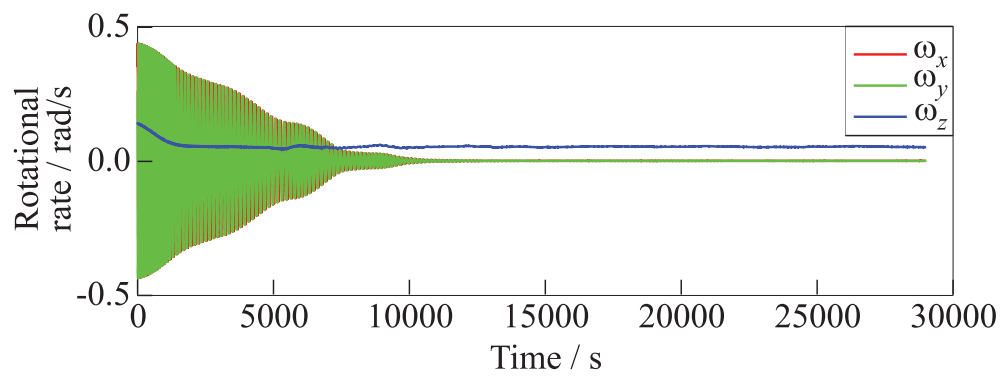

(a)

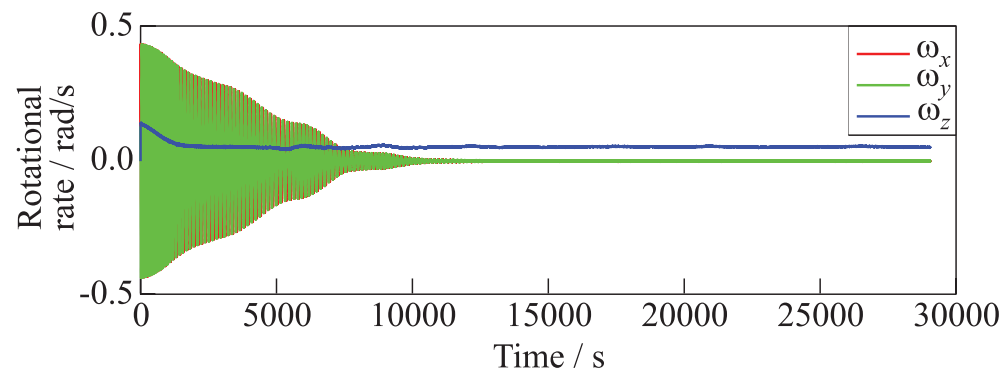

(b)

Figure 5 Modified Bdot. Detumble and spin rates of the $x$ axis are achieved: $(a)$ body rotational rates with regard to inertial frame; and $(b)$ estimated body rates. 


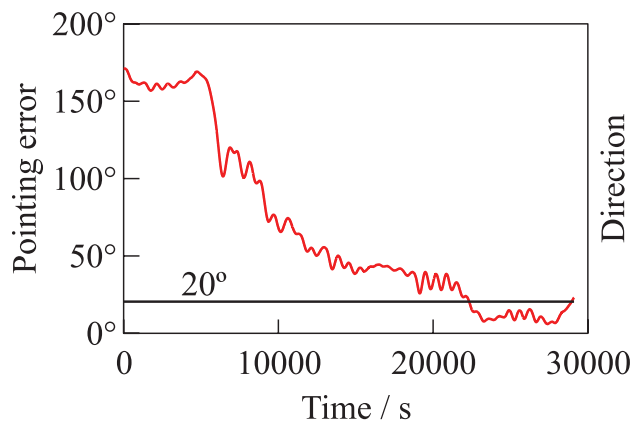

(a)

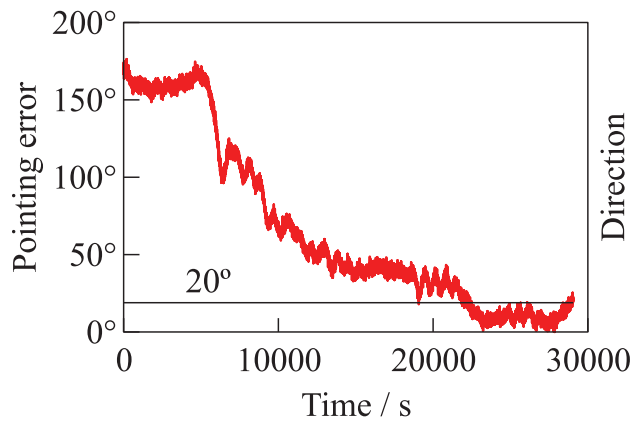

(b)

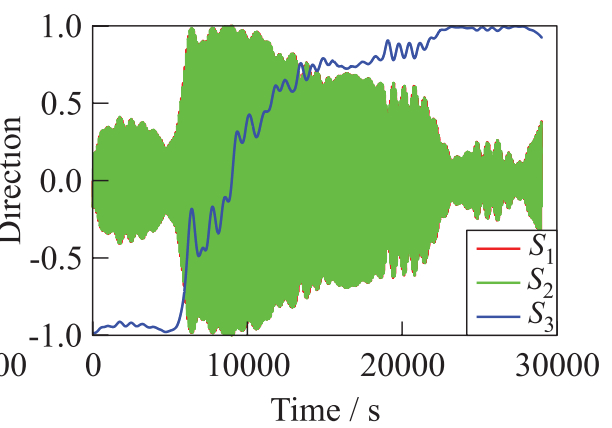

(c)

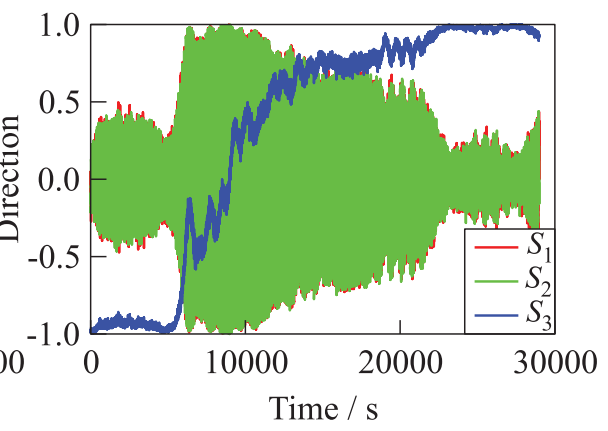

(d)

Figure 6 Coarse Sun pointing; the satellite succeeds in acquiring Sun pointing: (a) Sun pointing accuracy; (b) Sun pointing accuracy as measured; (c) direction of Sun in $B$; and $(d)$ direction of Sun in $B$ as measured.

Figure 6 shows that the CSP control can bring and maintain the satellite within acceptable bounds. From the direction of the Sun in body frame, it is clear that the satellite is rotating around the $z$ axis.

The FSP mode performances are depicted in Fig. 7. In this simulation, the angular velocity is acquired using noisy gyros. Compared to the results of the CSP modes, the achieved Sun pointing accuracy is more stable, which is the main advantage of the FSP mode over the CSP mode.

For comparison, the same mode is simulated using estimated angular rates, and the results are plotted in Fig. 8. Note that in this figure, the total time simulated is more than twice as long as in Fig. 7. Once Sun pointing is achieved within $20^{\circ}$, the pointing is stable and a failed gyroscope will thus only cause a larger initial acquisition time. 

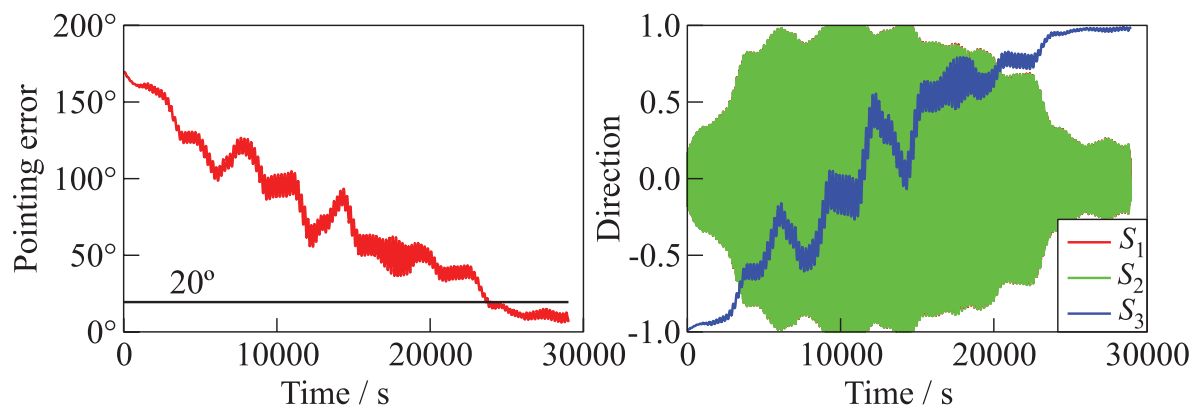

(a)

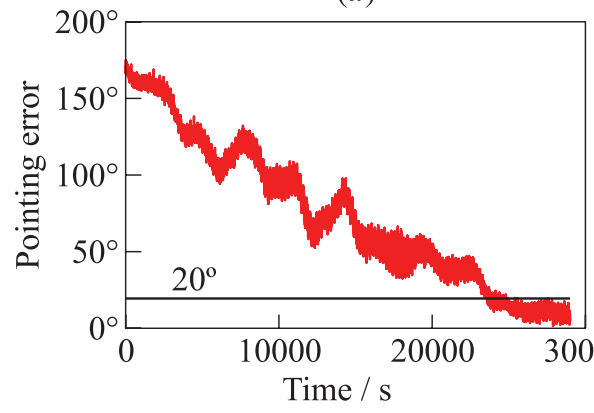

(b) (c)

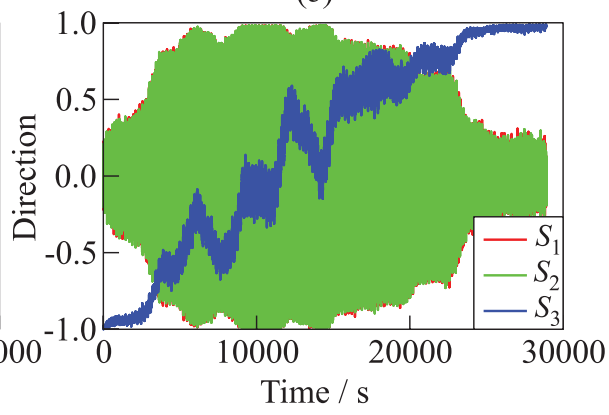

(d)

Figure 7 Fine Sun pointing; Sun pointing is achieved; gyros are used to measure the angular rates: $(a)$ Sun pointing accuracy; $(b)$ Sun pointing accuracy as measured; (c) direction of Sun in $B$; and $(d)$ direction of Sun in $B$ as measured.

\section{CONCLUDING REMARKS}

Developments of the attitude control system of the Delfi-n3xt nanosatellite were presented in this work. The attitude control system is designed such as to provide control redundancy between the reaction wheels and the MTQ. Keeping in mind the limited computational capability, simplicity was a design rule.

The proposed control modes use measurements from the sensors, e.g., the Sun sensor, the MGM, and the gyros. When information is missing, for instance on the attitude and the angular rates, simple estimation algorithms are designed based on the LoS measurements. For Sun pointing purposes, a quaternion estimated only from the measured Sun vector is sufficient. Apart from the gyros, the various sensor models implement realistic types and magnitudes for the errors. Extensive simulations were run that illustrate the good Sun pointing 


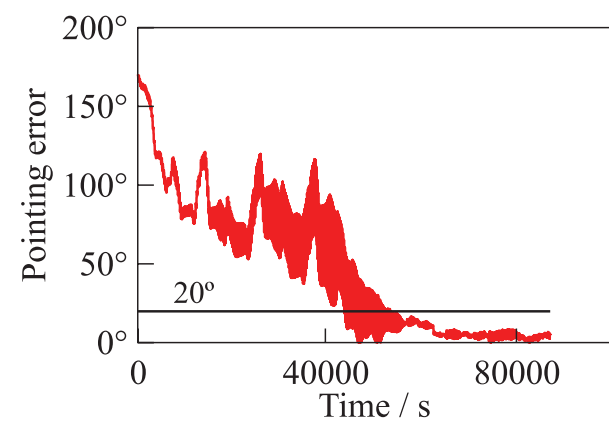

(a)

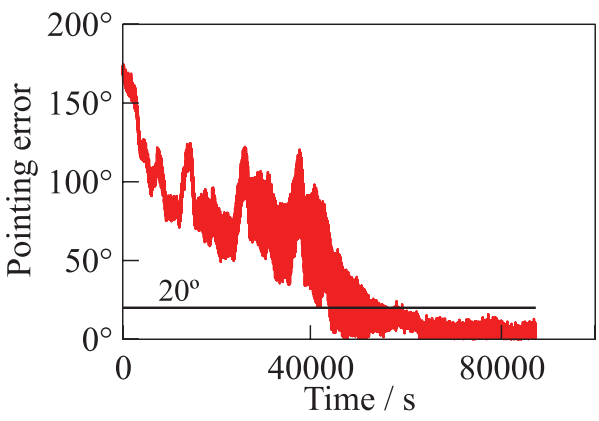

(b)

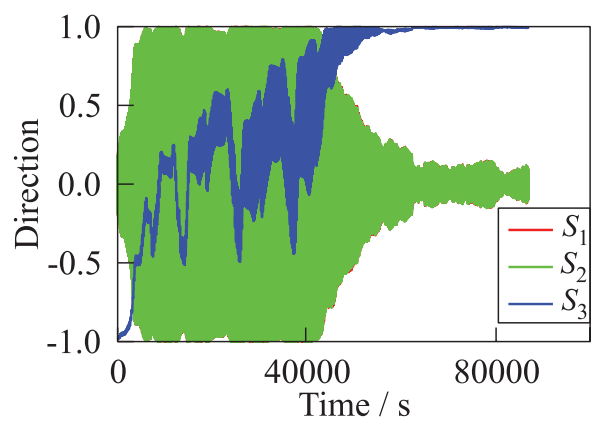

(c)

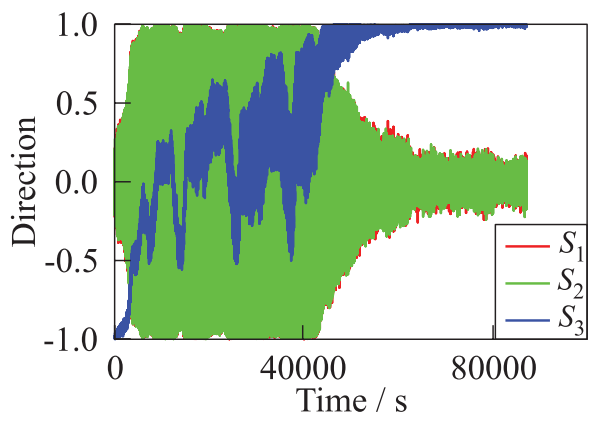

(d)

Figure 8 Fine Sun pointing; Sun pointing is achieved; angular rates are estimated: (a) Sun pointing accuracy; (b) Sun pointing accuracy as measured; $(c)$ direction of Sun in $B$; and $(d)$ direction of Sun in $B$ as measured.

performances of the proposed controllers in the presence of noises and disturbances.

\section{ACKNOWLEDGMENTS}

This research was supported by the TU Delft Delfi Satellite Program and by The Israel Science Foundation (Grant No. 1546/08).

\section{REFERENCES}

1. Creamer, G. 1999. The HESSI magnetic attitude control system. AIAA Guidance, Navigation, and Control Conference. 
2. Psiaki, M. L. 2000. Magnetic torquer attitude control via asymptotic periodic linear quadratic regulation. J. Guidance Control Dyn. 24.

3. Sedlund, C. A. 2008. A simple Sun-pointing magnetic controller for satellites in equatorial orbits. IEEEAC. Paper 1502.

4. Thienel, J., R. Bruninga, R. Stevens, C. Ridge, and C. Healy. 2009. The magnetic attitude control system for the Parkinson satellite (PSAT): A US Naval Academy Designed CubeSat. AIAA Paper No. 2009-5947.

5. Verbin, D., and S. Mualem. 2011. Minimal configuration attitude control for nanosatellite. Israel Annual Conference on Aeronautical Sciences. Tel-Aviv, Israel.

6. Stickler, A. C., and K. T. Alfriend. 1976. Elementary magnetic attitude control system. AIAA J. Spacecraft Rockets 13.

7. Wie, B., H. Weiss, and A. Arapostathis. 1989. Quaternion feedback regulators for spacecraft eigenaxis rotation. J. Guidance Control Dyn. 12(3):375-80.

8. Forbes, J.R., and C. J. Damaren. 2010. Geometric approach to spacecraft attitude control using magnetic and mechanical actuation. J. Guidance Control Dyn. 33(2):590-95.

9. Azor, R., I. Y. Bar-Itzhack, and R. R. Harman. 1998. Satellite angular rate estimation from vector measurements. J. Guidance Control Dyn. 21(3):450-57. 